\title{
BMJ Open Effect of vegetable consumption on the association between peripheral leucocyte telomere length and hypertension: a case-control study
}

\author{
Fuzhi Lian, ${ }^{1}$ Jinquan Wang, ${ }^{2}$ Xianhong Huang, ${ }^{1}$ Yinyin Wu, ${ }^{1}$ Yifei Cao, ${ }^{1}$ \\ Xiaohua Tan, ${ }^{1}$ Xianrong Xu, ${ }^{1}$ Yu Hong, ${ }^{1}$ Lei Yang, ${ }^{1,2}$ Xiang $\mathrm{Gao}^{3}$
}

To cite: Lian F, Wang J, Huang $X$, et al. Effect of vegetable consumption on the association between peripheral leucocyte telomere length and hypertension: a case-control study. BMJ Open 2015;5:e009305. doi:10.1136/bmjopen-2015009305

\section{- Prepublication history} and additional material is available. To view please visit the journal (http://dx.doi.org/ 10.1136/bmjopen-2015009305).

Received 5 July 2015 Revised 8 October 2015 Accepted 16 October 2015

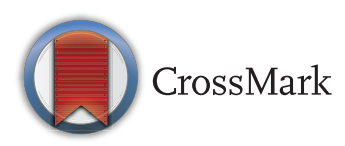

${ }^{1}$ School of Medicine, Hangzhou Normal University, Hangzhou, Zhejiang Province, China

${ }^{2}$ School of Life Science, Shihezi University, Shihezi, Xinjiang Uighur Autonomous Region, China

${ }^{3}$ Department of Nutritional Sciences, The Pennsylvania State University, University Park, Pennsylvania, USA

Correspondence to Dr Fuzhi Lian; Fuzhi.Lian@hznu.edu.cn and Dr Xiang Gao; xxg14@psu.edu

\section{ABSTRACT \\ Objectives: Peripheral leucocyte telomere length has been suggested to be inversely associated with hypertension risk. Both telomere length and hypertension risk can be modified by certain dietary factors, such as fruit and vegetables. This study was to examine the potential effect of these dietary factors on the association between telomere length and hypertension risk.}

Study design: A community-based case-control study.

Participants: 271 hypertensive patients and 455 normotensive controls aged $40-70$ years and living in Yinzhou, Zhejiang Province, China.

Outcome measures: Peripheral leucocyte relative telomere length (RTL) was measured using quantitative real-time PCR. Dietary intake was assessed by a brief semiquantitative food frequency questionnaire. The association between RTL and hypertension risk was analysed using logistic regression, and the modulatory effect of dietary intake on RTL-related hypertension risk was analysed using likelihood ratio tests.

Results: Among controls, longer age-adjusted RTL was associated with higher vegetable intake $(p=0.01)$. Individuals with longer age-adjusted RTL (based on median value) were $30 \%$ less likely to have hypertension ( $\mathrm{OR}=0.70,95 \% \mathrm{Cl} 0.52$ to $0.96 ; \mathrm{p}=0.03$ ). The observed RTL-hypertension relationship appeared to be modified by vegetable intake-longer RTL was significantly associated with lower hypertension risk only in those with greater vegetable consumption (OR=0.28, $95 \% \mathrm{Cl} 0.14$ to $0.57 ; p<0.001$ ), but not in those with lower vegetable intake

(P-interaction=0.008).

Conclusions: Certain dietary factors might modify telomere-related hypertension risk.

Telomeres are tandem repeat nucleotide sequences of TTAGGG that extend several kilobases at the end of mammalian chromosomes and play essential roles in the maintenance of chromosome stability and integrity. ${ }^{1}$ In somatic cells, telomeres shorten with mitotic cycles due to the so-called 'end-

\section{Strengths and limitations of this study}

- To the best of our knowledge, this is the first population-based study to examine the potential effects of food on the association between telomere length and hypertension risk.

- The study found that longer telomere length was associated with lower risk of hypertension only in those consuming the highest levels of vegetables, suggesting a synergistically protective effect on hypertension risk.

- The study used a brief version of a Chinese food frequency questionnaire that included only 11 food items, which limited our further analysis of the relationship between telomere length and specific nutrient intake.

replication problem' and also with oxidative stress. Therefore, telomere length is regarded as a marker for biological ageing and age-related diseases that reflects cell replicative history and lifetime cumulative oxidative stress from environmental exposure to cigarette smoking, poor nutrition and chronic inflammation. ${ }^{1-5}$ Essential hypertension is an ageing-related chronic pathological condition which has been considered an important cause of morbidity and mortality around the world. ${ }^{6}$ Animal studies found that telomerasedeficient mice with critical short telomeres developed higher blood pressure, which was associated with increased levels of circulating endothelin-1 and enhanced production of reactive oxygen species, ${ }^{7}$ suggesting that telomere attribution may have an important role in hypertension aetiology. In humans, it was also shown that telomere length in peripheral leucocytes was inversely associated with pulse pressure, ${ }^{8} 9$ diastolic blood pressure ${ }^{10}$ and hypertension risk. ${ }^{11-14}$

It has been suggested that leucocyte telomere length could be modulated by certain 
dietary factors. ${ }^{15} 16$ Individuals following a healthy lifestyle, including a higher intake of fruit and vegetables, had a longer peripheral leucocyte telomere length. ${ }^{17-19}$ In particular, previous studies showed that telomere length was positively associated with vegetable consumption $^{2021}$ and dietary intake of nutrients such as dietary fibre, ${ }^{22}$ vitamin $\mathrm{A}$ and $\beta$-carotene, ${ }^{21}{ }^{23}$ and vitamin $\mathrm{C} .{ }^{23}$ High consumption of fruit and vegetables was also related to lower risk of hypertension, ${ }^{24-26}$ whereas interventions with diets rich in fruit and vegetables that were reported to decrease blood pressure ${ }^{27} 28$ and the dietary approaches to stop hypertension (DASH) diet that was characterised with high intake of fruit and vegetables have been recommended as practical approaches for hypertension prevention and management by the American Heart Association. ${ }^{29}$ Since fruit and vegetables could be associated with both hypertension risk and telomere length, we hypothesised that these dietary factors may affect the relationship between telomere length and hypertension.

In the present study, we examined the association between telomere length and hypertension risk, the association between dietary factors and telomere length, and the potential modification of certain dietary factors (ie, consumption of fruit and vegetables) on the telomere-related hypertension risk in a case-control study of hypertension.

\section{MATERIALS AND METHODS}

Participants

The community-based case-control study was conducted in Yinzhou, Zhejiang Province, China. Participants were community residents who enrolled in the New Rural Cooperative Medical Insurance and came to local Community Health Centers for biennial routine health examination between April and July 2013. Hypertensive patients were invited to participate in this study when they met the following criteria: (1) previously diagnosed with hypertension, (2) at least two medical records of systolic blood pressure $\geq 140 \mathrm{~mm} \mathrm{Hg}$ and/or diastolic blood pressure $\geq 90 \mathrm{~mm} \mathrm{Hg}$, (3) on antihypertensive medication and (4) aged 40-70 years. Those with conditions of diabetes mellitus, dyslipidaemia, secondary hypertension, renal disease or a history of stroke and myocardial infarction were excluded. We then selected controls matched by age and gender. The controls had to meet the following criteria: (1) never diagnosed with hypertension, (2) not receiving antihypertensive treatment and (3) systolic blood pressure $<140 \mathrm{~mm} \mathrm{Hg}$ and diastolic blood pressure $<90 \mathrm{~mm} \mathrm{Hg}$ when measured at the time of health examination. Written informed consent was obtained from all study participants.

\section{Measurement of leucocyte relative telomere length}

At the time of visiting health centres, a venous blood sample was obtained from the antecubital vein for measurement of blood lipid levels and genomic DNA extraction. Genomic DNA was extracted from blood samples using TIANamp Blood DNA Kits (Tiangen Biotech, Beijing, China) and adjusted to a final concentration of $20 \mathrm{ng} / \mu \mathrm{L}$ with $10 \mathrm{mmol} / \mathrm{L}$ Tris-EDTA $(\mathrm{pH}$ 8.0) buffer. All DNA samples were checked for integrity by electrophoresis on $1.0 \%$ agarose gel and OD260/280 ratio (1.7-1.9). Relative telomere length (RTL) was measured with a quantitative real-time PCR method that compared the ratio of the telomere repeat copy number with the copy number of the $36 \mathrm{~B} 4$ single-copy gene in a given sample. ${ }^{30}$ The PCR reactions were performed in an ABI Prism 7000 Sequence Detection System. The primer sequences used for the telomeres were: forward primer: 5'-CGG TTT GTT TGG GTT TGG GTT TGG GTT TGG GTT TGG GTT-3', reverse primers: 5'-GGC TTG CCT TAC CCT TAC CCT TAC CCT TAC CCT TAC CCT- $3^{\prime}$. The primers for the single-copy gene 36B4 were: forward primer: 5'-CAG CAA GTG GGA AGG TGT AAT CC-3', reverse primer: 5'-CCC ATT CTA TCA TCA ACG GGT ACA A-3'. Twenty nanogram of genomic DNA was used for each amplification reaction. The thermal cycling profiles for both amplicons began with $95^{\circ} \mathrm{C}$ incubation for $15 \mathrm{~min}$, followed by 30 cycles of $15 \mathrm{~s}$ at $95^{\circ} \mathrm{C}$ and $30 \mathrm{~s}$ at $60^{\circ} \mathrm{C}$. The specificity of amplifications was determined by melting curve analysis. All reactions were done in 96-well PCR plates. Individual samples were measured in triplicate. A pooled DNA sample from 100 randomly selected participants was used as a reference and serially diluted (20,6.667, 2.222, 0.741 and $0.247 \mathrm{ng}$ ) to generate a fresh standard curve for each plate. For each sample, the quantity of telomere repeats and the quantity of $36 \mathrm{~B} 4$ copies were determined in comparison to the reference sample. The interbatch variability (coefficient of variation) in the study ranged from $4.9 \%$ to $12.3 \%$, which was consistent with previous reports. ${ }^{5}$

\section{Assessment of dietary intake and other potential confounders}

Dietary intake was assessed via a brief semiquantitative food frequency questionnaire. All participants were asked the amount and frequency of the consumption of 11 major food groups/items during the past 12 months: vegetables, fruit, egg, meat, poultry, fish/seafood, milk, soya milk, fried food, dessert and salt. The intake of salt was self-estimated based on gram per day (see online supplementary material).

During the survey, all participants were also interviewed with a brief questionnaire regarding alcohol consumption, smoking and physical activity (see online supplementary material). Participants who smoked at least one cigarette per week and drank at least once per week were defined as current smokers and current drinkers, respectively. Since only a few people were former smokers $(n=23)$ and former drinkers $(n=31)$, they were counted as non-smokers or non-drinkers. Those exercising at least twice a week were defined as exercisers. 
Weight, height, waist circumference and blood pressure were measured using standardised methods during the interview. Plasma concentrations of total cholesterol, triglycerides, low-density lipoprotein (LDL) cholesterol and high-density lipoprotein (HDL) cholesterol were measured on a Hitachi 7180 biochemistry automatic analyser (Hitachi High-Technologies Corporation, Minato-ku, Tokyo, Japan) as indicated in the manual.

\section{Statistical methods}

Statistical analysis was performed with SPSS V.16.0.1 (SPSS Inc, Chicago, Illinois, USA). Numeric values were presented as the mean (SD), and category values were expressed as the count (frequency) unless otherwise indicated. RTL was log-transformed to ensure the data fit normal distribution (one sample KolmogorovSmirnov test, $\mathrm{p}=0.20$ ). The log-transformed RTL was further age-adjusted using a residuals method. ${ }^{31}$ Log-transformed RTL was regressed on age, and age-adjusted RTL was computed by subtracting predicted value from log-transformed RTL. Independent $\mathrm{t}$ test or $\chi^{2}$ test was used for comparison of parameters, such as age, gender, body mass index and lipid levels, and for comparison between hypertensive and normotensive groups. Independent t test or one-way analysis of variance was used to compare the difference in age-adjusted RTL between genders and among various intake levels of dietary factors. Logistic regression was used to relate telomere length or dietary intake to hypertension risk, after adjustment for potential confounders including age, gender, body mass index, blood total cholesterol, triglycerides, HDL cholesterol, physical activity, alcohol drinking and cigarette smoking. Telomere length was categorised to short and long based on the median value of age-adjusted RTL.

To explore whether the relationship between RTL and hypertension was modified by dietary intake, we used likelihood ratio tests to compare nested models with or without the cross product between RTL and the covariates of interest. Subgroup analyses were then conducted when we observed significant interaction. A value of $\mathrm{p}<0.05$ was considered statistically significant.

\section{RESULTS}

During the recruitment period, a total of 1866 participants (1059 normotensive and 807 hypertensive patients) aged 40-70 years completed the survey and blood sample collection. After excluding 534 hypertensive patients with other conditions such as diabetes mellitus or dyslipidaemia, 277 cases were selected for final analysis. 458 controls were recruited from 709 normotensive subjects without listed chronic diseases and matched by age and gender. After further excluding 9 participants (6 cases and 3 controls) who had poor measurements of telomere length, a total of 726 individuals (271 cases and 455 controls) with complete data and acceptable DNA quality were included in the analysis (table 1).

Table 1 Characteristics of participants, according to hypertension status

\begin{tabular}{|c|c|c|c|}
\hline & Normotensive $(n=455)$ & Hypertensive $(n=271)$ & p Value \\
\hline Gender & & & 0.66 \\
\hline Male & $231(50.8)$ & $133(49.1)$ & \\
\hline Female & 224 (49.2) & 138 (50.9) & \\
\hline Age (years) & $56.4(7.51)$ & $57.5(7.97)$ & 0.067 \\
\hline Regular exercise & & & 0.61 \\
\hline No & 309 (67.9) & $189(69.7)$ & \\
\hline Yes & $146(32.1)$ & $82(30.3)$ & \\
\hline Current alcohol consumption & & & 0.51 \\
\hline No & $281(61.8)$ & $174(64.2)$ & \\
\hline Yes & $174(38.2)$ & $97(35.8)$ & \\
\hline Current smoking & & & 0.014 \\
\hline No & $303(66.6)$ & $204(75.3)$ & \\
\hline Yes & $152(33.4)$ & $67(24.7)$ & \\
\hline Body mass index $\left(\mathrm{kg} / \mathrm{m}^{2}\right)$ & $22.0(2.52)$ & $23.2(3.04)$ & $<0.001$ \\
\hline Waist circumference $(\mathrm{cm})$ & $78.5(7.46)$ & $81.5(8.26)$ & $<0.001$ \\
\hline Systolic blood pressure $(\mathrm{mm} \mathrm{Hg})$ & $120(11)$ & $148(12.56)$ & $<0.001$ \\
\hline Diastolic blood pressure $(\mathrm{mm} \mathrm{Hg})$ & $74.6(7.49)$ & $90.2(10.7)$ & $<0.001$ \\
\hline Total cholesterol (mmol/L) & $4.34(0.51)$ & $4.36(0.51)$ & 0.62 \\
\hline Triglycerides (mmol/L) & $1.07(0.33)$ & $1.15(0.39)$ & $<0.01$ \\
\hline HDL cholesterol (mmol/L) & $1.38(0.26)$ & $1.35(0.23)$ & 0.074 \\
\hline LDL cholesterol (mmol/L) & $2.62(0.48)$ & $2.67(0.46)$ & 0.24 \\
\hline RTL† & $1.05(0.80,1.34)$ & $0.98(0.70,1.31)$ & - \\
\hline Age-adjusted RTL & $0.069(0.984)$ & $-0.117(1.015)$ & 0.015 \\
\hline
\end{tabular}


There was no significant difference in gender and age between the case and control groups (table 1). Hypertensive patients had a significantly higher prevalence of smoking, and higher waist circumference, body mass index, systolic and diastolic blood pressure, blood triglycerides and LDL cholesterol than normotensive controls. Age was inversely associated with RTL (coefficient $\beta=-0.002, p=0.015)$. Further, the hypertensive group had shorter age-adjusted RTL than the normotensive group (mean age-adjusted RTL: -0.117 vs 0.069 , $\mathrm{p}=0.015$ ) (table 1). There was no significant difference in age-adjusted RTL between males and females in the case and control groups.

Since hypertension had a significant impact on telomere length, we examined the effect of dietary factors on telomere length only in the control group. In normotensive controls, higher vegetable intake was associated with longer age-adjusted RTL $(p=0.01)$, while regular consumption of soya milk $(\mathrm{p}=0.01)$ and regular consumption of fried food $(p=0.045)$ were associated with shorter telomere length. We did not observe significant associations between other dietary factors (fruit, meat, poultry, fish/seafood, milk, dessert and salt) and telomere length (table 2).

As expected, longer age-adjusted telomere length was associated with decreased risk of hypertension $(\mathrm{OR}=0.70,95 \%$ CI 0.52 to $0.96 ; \mathrm{p}=0.03)$, after adjustment for potential confounders (table 3). We also observed a trend between greater consumption of vegetables, but not fruit, and lower prevalence of hypertension (table 3). For other dietary factors, individuals with a greater intake of salt or a lower intake of fish/ seafood had a higher likelihood of having hypertension (table 3).

We further examined whether the association between telomere length and hypertension was modified by fruit and vegetables. We observed that longer RTL was only significantly associated with lower hypertension risk in those with greater vegetable consumption $(\mathrm{OR}=0.28$, $95 \%$ CI 0.14 to $0.57 ; \mathrm{p}<0.001)$, but not in those with lower vegetable intake (P-interaction $=0.008) \quad$ (table 4$)$. The significance remained even after Bonferroni correction for multiple comparisons $(\alpha=0.05 / 2)$. In contrast, the interaction between RTL and fruit was not significant (P-interaction $>0.2$ ). Similar non-significant results were also observed for other dietary factors (P-interaction $>0.1$ for all $)$.

\section{DISCUSSION}

In this community-based case-control study, we found that longer telomere length in peripheral leucocytes was associated with $30 \%$ lower odds of having hypertension, and this association was more pronounced among participants with a higher intake of vegetables. To the best of our knowledge, this is the first study to show that certain dietary factors may modify the association between telomeres and hypertension risk.
Table 2 Effect of intake levels of major dietary factors on age-adjusted relative telomere length among 455 normotensive controls*

\begin{tabular}{|c|c|c|c|}
\hline Dietary factors & n (\%) & $\begin{array}{l}\text { Age-adjusted } \\
\text { RTL (SD) }\end{array}$ & p Value† \\
\hline \multicolumn{4}{|c|}{ Vegetables (g/day) } \\
\hline$<50$ & $72(15.9)$ & $-0.064(0.94)$ & 0.01 \\
\hline $50-150$ & $276(60.8)$ & $0.011(0.99)$ & \\
\hline$>150$ & $106(23.3)$ & $0.32(0.98) \ddagger$ & \\
\hline \multicolumn{4}{|l|}{ Fruit (g/day) } \\
\hline$<50$ & $141(31.1)$ & $0.17(0.97)$ & 0.35 \\
\hline $50-150$ & $263(57.9)$ & $0.031(0.98)$ & \\
\hline$>150$ & $50(11.0)$ & $0.010(1.08)$ & \\
\hline \multicolumn{4}{|l|}{ Meat (g/week) } \\
\hline$<100$ & $240(52.7)$ & $0.093(0.92)$ & 0.58 \\
\hline $100-200$ & $148(32.5)$ & $0.083(1.02)$ & \\
\hline$>200$ & $67(14.7)$ & $-0.046(1.11)$ & \\
\hline \multicolumn{4}{|c|}{ Fish/seafood (g/week) } \\
\hline$<100$ & $217(47.7)$ & $0.16(1.00)$ & 0.17 \\
\hline $100-200$ & $128(28.1)$ & $0.017(0.95)$ & \\
\hline$>200$ & $110(24.2)$ & $-0.044(0.99)$ & \\
\hline \multicolumn{4}{|l|}{ Poultry (g/week) } \\
\hline$<50$ & $126(27.7)$ & $0.21(0.96)$ & 0.16 \\
\hline $50-100$ & $277(60.9)$ & $0.019(0.96)$ & \\
\hline$>100$ & $52(11.4)$ & $-0.005(1.12)$ & \\
\hline \multicolumn{4}{|l|}{ Milk } \\
\hline None & $287(63.4)$ & $0.13(1.00)$ & 0.07 \\
\hline Regularly & $166(36.6)$ & $-0.04(0.95)$ & \\
\hline \multicolumn{4}{|l|}{ Soya milk } \\
\hline None & $331(73.1)$ & $0.15(0.96)$ & 0.005 \\
\hline Regularly & $122(26.9)$ & $-0.15(1.03)$ & \\
\hline \multicolumn{4}{|l|}{ Egg (/week) } \\
\hline None & $104(22.9)$ & $-0.007(1.02)$ & 0.07 \\
\hline $1-4$ & $295(65.0)$ & $0.21(0.91)$ & \\
\hline$>4$ & $55(12.1)$ & $0.093(0.92)$ & \\
\hline \multicolumn{4}{|l|}{ Fried food } \\
\hline None & $178(39.2)$ & $0.19(0.97)$ & 0.045 \\
\hline Regularly & $276(60.8)$ & $-0.002(0.99)$ & \\
\hline \multicolumn{4}{|l|}{ Dessert } \\
\hline None & $143(31.5)$ & $0.15(0.95)$ & 0.28 \\
\hline Regularly & $311(68.5)$ & $0.038(1.00)$ & \\
\hline \multicolumn{4}{|l|}{ Salt (g/day) } \\
\hline$<6$ & 341 (74.9) & $0.08(0.92)$ & 0.10 \\
\hline $6-12$ & $100(22.0)$ & $0.11(1.14)$ & \\
\hline$>12$ & $14(3.1)$ & -0.48 & \\
\hline \multicolumn{4}{|c|}{$\begin{array}{l}{ }^{*} \text { No significant } P \text { for trend was found for any of the analyses. } \\
+ \text { Independent } t \text { test or one-way analysis of variance. } \\
\neq p<0.05 \text { in comparison to vegetable intake of }<50 \text { and } \\
50-100 \text { g/day. }\end{array}$} \\
\hline
\end{tabular}

The finding that hypertensive patients had shorter peripheral telomere length than healthy controls was in agreement with previous reports. ${ }^{311-13}{ }^{32}$ For example, Lung $e t a l^{11}$ showed that the white blood cell telomere length of 73 adults with hypertension was shorter than that of 76 healthy controls in a community of southern Taiwan, and Yang et $a l^{12}$ found that 388 hypertensive patients had a shorter leucocyte telomere length than 379 normotensive controls in central China. Similarly, one cohort study also showed that shorter telomere 
Table 3 Adjusted ORs and 95\% Cls of hypertension, according to age-adjusted relative telomere length (RTL) and dietary intake*

\begin{tabular}{|c|c|c|c|c|c|}
\hline Variables & $\begin{array}{l}\text { Normotensive } \\
\text { n (\%) }\end{array}$ & $\begin{array}{l}\text { Hypertensive } \\
\text { n (\%) }\end{array}$ & OR (95\% Cl) & P-difference & P-trend \\
\hline Age-adjusted RTL & & & & & - \\
\hline Short & $212(46.6)$ & $151(55.7)$ & 1.00 (reference) & & \\
\hline Long & $243(53.4)$ & $120(44.3)$ & 0.70 (0.52 to 0.96$)$ & 0.03 & \\
\hline Vegetables (g/day) & & & & & 0.09 \\
\hline$<50$ & $72(15.9)$ & $56(20.7)$ & 1.00 (reference) & & \\
\hline $50-150$ & $276(60.8)$ & $161(59.4)$ & $0.72(0.48$ to 1.09$)$ & 0.12 & \\
\hline$>150$ & $106(23.3)$ & 54 (19.9) & 0.65 (0.40 to 1.06$)$ & 0.08 & \\
\hline \multicolumn{6}{|l|}{ Fruit (g/day) } \\
\hline$<50$ & $141(31.1)$ & $83(30.6)$ & 1.00 (reference) & & 0.80 \\
\hline $50-150$ & $263(57.9)$ & $156(57.6)$ & 0.97 (0.69 to 1.37$)$ & 0.88 & \\
\hline$>150$ & $50(11.0)$ & $32(11.8)$ & 1.05 (0.61 to 1.78$)$ & 0.87 & \\
\hline Meat (g/week) & & & & & 0.60 \\
\hline$<100$ & $240(52.7)$ & $147(54.2)$ & 1.00 (reference) & & \\
\hline $100-200$ & $148(32.5)$ & $88(32.5)$ & 0.97 (0.69 to 1.36$)$ & 0.84 & \\
\hline$>200$ & 67 (14.7) & $36(13.3)$ & 0.90 (0.57 to 1.43$)$ & 0.65 & \\
\hline Fish/seafood (g/week) & & & & & 0.035 \\
\hline$<100$ & $217(47.7)$ & $150(55.4)$ & 1.00 (reference) & & \\
\hline $100-200$ & $128(28.1)$ & $70(25.8)$ & 0.81 (0.56 to 1.16$)$ & 0.25 & \\
\hline$>200$ & $110(24.2)$ & $51(18.8)$ & $0.63(0.42$ to 0.95$)$ & 0.03 & \\
\hline Poultry (g/week) & & & & & 0.83 \\
\hline$<50$ & $126(27.7)$ & $68(25.1)$ & 1.00 (reference) & & \\
\hline $50-100$ & 277 (60.9) & $181(66.8)$ & 1.21 (0.84 to 1.72$)$ & 0.31 & \\
\hline$>100$ & $52(11.4)$ & $22(8.1)$ & 0.76 (0.42 to 1.38$)$ & 0.37 & \\
\hline Milk & & & & & - \\
\hline None & 287 (63.4) & 177 (65.6) & 1.00 (reference) & & \\
\hline Regularly & $166(36.6)$ & 93 (34.4) & 0.89 (0.64 to 1.23$)$ & 0.47 & \\
\hline \multicolumn{6}{|l|}{ Soya milk } \\
\hline None & $331(73.1)$ & $202(75.1)$ & 1.00 (reference) & & - \\
\hline Regularly & $122(26.9)$ & 67 (24.9) & 0.92 (0.65 to 1.31$)$ & 0.92 & \\
\hline Egg (/week) & & & & & 0.56 \\
\hline None & $104(22.9)$ & $62(23)$ & 1.00 (reference) & & \\
\hline $1-4$ & $295(65.0)$ & $168(62.2)$ & 0.87 (0.60 to 1.27$)$ & 0.48 & \\
\hline$\geq 5$ & 55 (12.1) & 40 (14.8) & 1.07 (0.63 to 1.82$)$ & 0.80 & \\
\hline Fried food & & & & & - \\
\hline None & $178(39.2)$ & 95 (35.2) & 1.00 (reference) & & \\
\hline Regularly & $276(60.8)$ & $175(64.8)$ & 1.14 (0.83 to 1.57$)$ & 0.42 & \\
\hline Dessert & & & & & - \\
\hline None & $143(31.5)$ & 79 (29.2) & 1.00 (reference) & & \\
\hline Regularly & $311(68.5)$ & $192(70.8)$ & 1.09 (0.78 to 1.53$)$ & 0.60 & \\
\hline Salt (g/day) & & & & & $<0.001$ \\
\hline$<6$ & 341 (74.9) & $151(55.7)$ & 1.00 (reference) & & \\
\hline $6-12$ & $100(22.0)$ & $92(33.9)$ & 2.21 (1.55 to 3.14$)$ & $<0.001$ & \\
\hline$>12$ & $14(3.1)$ & $28(10.3)$ & 4.81 (2.43 to 9.52$)$ & $<0.001$ & \\
\hline
\end{tabular}

${ }^{*}$ Adjusted for age $(\mathrm{y})$, gender, body mass index $\left(\mathrm{kg} / \mathrm{m}^{2}\right)$, plasma total cholesterol, plasma high-density lipoprotein cholesterol, plasma triglycerides, exercise (2+ times per week, yes/no), current alcohol consumption (at least once a week, yes/no) and current smoking (yes/no).

length at the baseline was associated with increased risk of developing hypertension after 5 years of follow-up. ${ }^{12}$ Shorter telomere length was associated with vascular senescence, increased oxidative stress and chronic inflammation, ${ }^{1}$ which were all related to increased risk of hypertension. ${ }^{33}$ Telomerase-deficient mice exhibited the phenotype of progressive telomere shortening in successive generations. These mice developed higher blood pressure than wild-type mice, which was related to higher levels of circulating endothelin-1 and enhanced production of reactive oxygen species. ${ }^{7}$ Our results, together with previous studies, provide convincing evidence that shorter telomere length was associated with hypertension and may play an important role in the aetiology of hypertension.

In this study, we observed that greater intake of vegetables was related to longer peripheral telomere length, which is in accordance with previous reports that a healthy lifestyle including high consumption of fruit and vegetable was associated with longer telomere 
Table 4 Adjusted ORs and 95\% Cls of hypertension, according to longer age-adjusted relative telomere length (RTL), stratified by vegetable intake*

\begin{tabular}{lllllll}
\hline \multirow{2}{*}{ Vegetable intake } & Age-adjusted RTL & $\begin{array}{l}\text { Normotensive } \\
\mathbf{n}(\%)\end{array}$ & $\begin{array}{l}\text { Hypertensive } \\
\mathbf{n}(\%)\end{array}$ & OR (95\% Cl) & P-difference & P-interaction \\
\hline$<50$ g/day & Short & $36(50.0)$ & $33(58.9)$ & 1.00 (reference) & & 0.008 \\
& Long & $36(50.0)$ & $23(41.1)$ & $0.68(0.33$ to 1.40$)$ & 0.330 & \\
$50-150$ g/day & Short & $136(49.3)$ & $83(51.6)$ & $1.00($ reference) & & \\
& Long & $140(50.7)$ & $78(48.4)$ & $0.95(0.64$ to 1.42$)$ & 0.809 & \\
$>150$ g/day & Short & $39(36.8)$ & $35(64.8)$ & 1.00 (reference) & & \\
& Long & $67(63.2)$ & $19(35.2)$ & $0.28(0.14$ to 0.57$)$ & $<0.001$ & \\
\hline
\end{tabular}

${ }^{*}$ Adjusted for age $(\mathrm{y})$, gender, body mass index $\left(\mathrm{kg} / \mathrm{m}^{2}\right)$, plasma total cholesterol, plasma high-density lipoprotein cholesterol, plasma triglycerides, exercise (2+ times per week, yes/no), alcohol (at least once a week, yes/no) and current smoking (yes/no).

length. ${ }^{17-21}$ Vegetables usually contain relatively high levels of carotenoids, vitamin $\mathrm{C}$ and dietary fibre, the food components that were associated with the long telomere in many studies. ${ }^{21-23}$ Unfortunately, we were not able to further analyse the effect of specific nutrients or dietary components on telomere length due to the simple dietary questionnaires used in this study.

Interestingly, we observed that long telomere length was related to lower risk of hypertension only in those consuming the highest levels of vegetables. Our results might suggest a synergistically protective effect of vegetable intake in those with a longer telomere. This finding also highlights the importance of incorporating the dietary intervention into an integrated approach to slow down the process of biological ageing and prevent against hypertension. However, this phenomenon may also be partly explained by the fact that lower levels of vegetable intake by themselves are risk factors for hypertension, ${ }^{29}$ and the protective effect of a long telomere cannot be teased out due to limited statistical power. Consistent with this notion, Hou et $a b^{34}$ showed that a short telomere was associated with a higher risk of gastric cancer only in those without risk factors for gastric cancer; and Diaz et $a l^{35}$ found that healthy lifestyle behaviours modulated the association between telomere length and coronary artery calcium. In this context, more studies are warranted to elucidate the potential role of diet in the telomere-hypertension relationship.

The study had several limitations. First, telomere length was measured by the quantitative PCR method. The method usually has higher variation than that measured by southern blot (more than $10 \%$ in most references).$^{5}$ In this study, the coefficient of variation for different measurements of the same sample ranged from $10 \%$ to $20 \%$, which was consistent with previous reports. The limitation of the method was that it required a larger sample size to find significant results, and might mask existent weak correlations (eg, the effects of other foods on the telomere-hypertension relationship) in current samples. Second, the dietary questionnaire used in the study has not been validated. However, the association between intake of salt, vegetables, and fish and hypertension risk and/or telomere length provides indirect evidence of the validity of this questionnaire. Since there were only 11 food items in the questionnaire, we were not able to calculate total energy consumption for food intake adjustment. We were also unable to further analyse the relationship between telomere length and specific nutrients or food components, which could be of importance to understand the observed associations. Further, reverse causality is of concern due to the retrospective study design. Participants might switch to a healthier dietary pattern as a result of hypertension diagnosis/symptoms. This would lead to an underestimation for the potential effect of fruit/vegetables on telomere-hypertension relationship.

In summary, the study suggests that greater vegetable intake may modify the association between telomere length and hypertension. Further studies with a validated dietary questionnaire and prospective study design are warranted to replicate our observations.

Contributors FL, LY and $X G$ are responsible for the study concept and design, drafting of the manuscript and critical revision of the manuscript. JW and $Y C$ are responsible for the study design and measurement of RTL. XH, $Y W, F L$ and $X G$ are responsible for data analysis and interpretation. $X T, X X$ and $\mathrm{YH}$ are responsible for the participant recruitment and collection of medical information.

Funding The work was supported by the National Basic Research programme of China grant number 2012CB911200 and the programme for Zhejiang Leading Team of Science and Technology Innovation of China grant number 2011R50021.

Competing interests All authors have completed the ICMJE uniform disclosure form at http://www.icmje.org/coi_disclosure.pdf and declare: FL and LY had financial support from the National Basic Research Program of China and the Program for Zhejiang Leading Team of Science and Technology Innovation of China for the submitted work; no financial relationships with any organisations that might have an interest in the submitted work in the previous 3 years; no other relationships or activities that could appear to have influenced the submitted work.

Ethics approval The study was approved by the Hangzhou Normal University ethics committee.

Provenance and peer review Not commissioned; externally peer reviewed.

Data sharing statement No additional data are available.

Open Access This is an Open Access article distributed in accordance with the Creative Commons Attribution Non Commercial (CC BY-NC 4.0) license, which permits others to distribute, remix, adapt, build upon this work non- 
commercially, and license their derivative works on different terms, provided the original work is properly cited and the use is non-commercial. See: http:// creativecommons.org/licenses/by-nc/4.0/

\section{REFERENCES}

1. Aubert G, Lansdorp PM. Telomeres and aging. Physiol Rev 2008;88:557-79.

2. Houben JM, Moonen HJ, van Schooten FJ, et al. Telomere length assessment: biomarker of chronic oxidative stress? Free Radic Biol Med 2008;44:235-46.

3. Demissie S, Levy D, Benjamin EJ, et al. Insulin resistance, oxidative stress, hypertension, and leukocyte telomere length in men from the Framingham Heart Study. Aging Cell 2006;5:325-30.

4. Babizhayev MA, Savel'yeva EL, Moskvina SN, et al. Telomere length is a biomarker of cumulative oxidative stress, biologic age, and an independent predictor of survival and therapeutic treatment requirement associated with smoking behavior. Am J Ther 2011;18: e209-26.

5. Sanders JL, Newman AB. Telomere length in epidemiology: a biomarker of aging, age-related disease, both, or neither? Epidemiol Rev 2013;35:112-31.

6. Carretero OA, Oparil S. Essential hypertension. Part I: definition and etiology. Circulation 2000;101:329-35.

7. Perez-Rivero G, Ruiz-Torres MP, Rivas-Elena JV, et al. Mice deficient in telomerase activity develop hypertension because of an excess of endothelin production. Circulation 2006;114:309-17.

8. Benetos A, Okuda K, Lajemi M, et al. Telomere length as an indicator of biological aging: the gender effect and relation with pulse pressure and pulse wave velocity. Hypertension 2001;37(2 Pt 2):381-5.

9. Jeanclos E, Schork NJ, Kyvik KO, et al. Telomere length inversely correlates with pulse pressure and is highly familial. Hypertension 2000;36:195-200.

10. Fitzpatrick AL, Kronmal RA, Gardner JP, et al. Leukocyte telomere length and cardiovascular disease in the cardiovascular health study. Am J Epidemiol 2007;165:14-21.

11. Lung FW, Ku CS, Kao WT. Telomere length may be associated with hypertension. J Hum Hypertens 2008;22:230-2.

12. Yang $\mathrm{Z}$, Huang $\mathrm{X}$, Jiang $\mathrm{H}$, et al. Short telomeres and prognosis of hypertension in a chinese population. Hypertension 2009;53:639-45.

13. Bhupatiraju C, Saini D, Patkar S, et al. Association of shorter telomere length with essential hypertension in Indian population. Am J Hum Biol 2012;24:573-8.

14. Ma L, Li Y, Wang J. Telomeres and essential hypertension. Clin Biochem 2015. http://dx.doi.org/10.1016/j.clinbiochem.2015.07.0 [epub ahead of print]

15. Jennings BJ, Ozanne SE, Hales CN. Nutrition, oxidative damage, telomere shortening, and cellular senescence: individual or connected agents of aging? Mol Genet Metab 2000;71:32-42.

16. Paul L. Diet, nutrition and telomere length. J Nutr Biochem 2011;22:895-901.
17. Nettleton JA, Diez-Roux A, Jenny NS, et al. Dietary patterns, food groups, and telomere length in the Multi-Ethnic Study of Atherosclerosis (MESA). Am J Clin Nutr 2008;88:1405-12.

18. Mirabello L, Huang WY, Wong JY, et al. The association between leukocyte telomere length and cigarette smoking, dietary and physical variables, and risk of prostate cancer. Aging Cell 2009:8:405-13.

19. Sun Q, Shi L, Prescott J, et al. Healthy lifestyle and leukocyte telomere length in U.S. women. PLOS ONE 2012;7:e38374

20. Tiainen AM, Männistö S, Blomstedt PA, et al. Leukocyte telomere length and its relation to food and nutrient intake in an elderly population. Eur J Clin Nutr 2012;66:1290-4.

21. Marcon F, Siniscalchi E, Crebelli $R$, et al. Diet-related telomere shortening and chromosome stability. Mutagenesis 2012;27:49-57.

22. Cassidy A, De Vivo I, Liu Y, et al. Associations between diet, lifestyle factors, and telomere length in women. Am J Clin Nutr 2010;91:1273-80.

23. Xu Q, Parks CG, DeRoo LA, et al. Multivitamin use and telomere length in women. Am J Clin Nutr 2009;89:1857-63.

24. Miura K, Greenland P, Stamler J, et al. Relation of vegetable, fruit, and meat intake to 7-year blood pressure change in middle-aged men: the Chicago Western Electric Study. Am J Epidemiol 2004;159:572-80.

25. Dauchet L, Kesse-Guyot E, Czernichow S, et al. Dietary patterns and blood pressure change over 5-y follow-up in the SU.VI.MAX cohort. Am J Clin Nutr 2007;85:1650-6.

26. Boeing $\mathrm{H}$, Bechthold $\mathrm{A}$, Bub A, et al. Critical review: vegetables and fruit in the prevention of chronic diseases. Eur J Nutr 2012;51:637-63.

27. Singh RB, Sircar AR, Rastogi SS, et al. Dietary modulators of blood pressure in hypertension. Eur J Clin Nutr 1990;44:319-27.

28. Appel LJ, Moore TJ, Obarzanek E, et al. A clinical trial of the effects of dietary patterns on blood pressure. DASH Collaborative Research Group. N Engl J Med 1997;336:1117-24.

29. Appel LJ, Brands MW, Daniels SR, et al. Dietary approaches to prevent and treat hypertension: a scientific statement from the American Heart Association. Hypertension 2006;47:296-308.

30. Cawthon RM. Telomere measurement by quantitative PCR. Nucleic Acids Res 2002;30:e47.

31. Willett W. Nutritional Epidemiology. Oxford: Oxford Oxford University Press, 1998

32. Farrag W, Eid M, El-Shazly S, et al. Angiotensin II type 1 receptor gene polymorphism and telomere shortening in essential hypertension. Mol Cell Biochem 2011;351:13-18.

33. Dinh QN, Drummond GR, Sobey CG, et al. Roles of Inflammation, Oxidative Stress, and Vascular Dysfunction in Hypertension. Biomed Res Int 2014;2014:406960.

34. Hou L, Savage SA, Blaser MJ, et al. Telomere length in peripheral leukocyte DNA and gastric cancer risk. Cancer Epidemio Biomarkers Prev 2009;18:3103-9.

35. Diaz VA, Mainous AG III, Everett CJ, et al. Effect of healthy lifestyle behaviors on the association between leukocyte telomere length and coronary artery calcium. Am J Cardiol 2010;106:659-63. 